\title{
Mortality Pattern of In-Born vs Out-Born Neonates - Comparative Study of Neonates Admitted to Special New Born Care Units of a Tertiary Care Hospital of Hilly Region of North India
}

\author{
Ambika Sood ${ }^{1}$, Rakesh Sharma ${ }^{2}$, Raju Katoch ${ }^{3}$, Sanya Sharma ${ }^{4}$ \\ 1, 2, 3 Department of Paediatrics, Indira Gandhi Medical College, Shimla, Himachal Pradesh, India. \\ ${ }^{4}$ Dr. SLBS, GMC, Nerchowk, Mandi, Himachal Pradesh, India.
}

\section{ABSTRACT}

\section{BACKGROUND}

The high incidence of global neonatal mortality has triggered several efforts to improve facility-based neonatal care but despite these many advances in perinatal and neonatal care, the outcomes achievable at different levels are comparable. This study was undertaken to compare the mortality pattern among both the inborn and outborn neonates admitted to the special new born care units (SNCU)s of tertiary care hospital of Shimla city, in the hilly state of Himachal Pradesh.

\section{METHODS}

A cross-sectional study involving a record review of all neonates admitted to the specialized neonatal care units of the paediatric ward of IGMC, Shimla \& Kamla Nehru Hospital (KNH) over five years from January 2016 to December 2020 was conducted. The most important causes of mortality and its associated factors were analysed, and a statistical inference was made.

\section{RESULTS}

Among the 4018 outborn neonates admitted to the SNCU of the pediatric ward of IGMC, Shimla, 2440 (60.73 \%) were males and 1578 (39.27\%) were females while 6607 inborn neonates were admitted to the SNCU of KNH Shimla and among them 3655 (55.32 \%) were males and 2952 (44.68 \%) were females. In the outborn SNCU of IGMC Shimla, 307 (7.64\%) died while in the inborn SNCU of KNH Shimla, 366 $(5.54 \%)$ neonates died over a span of 5 years. In outborn SNCU of IGMC Shimla, a maximum of 110 (35.83\%) neonates died due to sepsis/pneumonia/meningitis, followed by $60(19.54 \%)$ due to respiratory distress syndrome and $50(16.29 \%)$ due to HIE/moderate-severe birth asphyxia while in case of inborn SNCU of KNH, maximum deaths of 115 (31.42\%) were due to respiratory distress syndrome, followed by 93 (25.41\%) due to sepsis/pneumonia/meningitis and 69 (18.85\%) due to HIE/moderate-severe birth asphyxia. At outborn SNCU of IGMC, Shimla, most of the neonates who died 118 (38.44 \%) weighed about 1500-2499 gm, while in inborn SNCU of KNH, Shimla, most of the neonates 147 (40.16\%) had weight between 1000-1499 gm. At outborn SNCU of IGMC Shimla, 131 (42.67 \%) were preterm while at inborn SNCU of KNH, 305 (83.3 \%) were preterm.

\section{CONCLUSIONS}

Sepsis, birth asphyxia and RDS are the important causes of mortality, which must be urgently addressed. Improving antenatal care, improved access to health facilities, early identification of danger signs, timely referral, capacity building, can reduce neonatal mortality.

\section{KEY WORDS}

Comparison, Neonatal Mortality, SNCUs, Tertiary Care Hospital, Inborn Neonate, Outborn Neonate.
Corresponding Author:

Dr. Ambika Sood, Set No. 7, Jasmine Villa, Near CPRI, Kanlog,

Shimla, Himachal Pradesh, India.

E-mail:drambikasood@gmail.com

DOI: $10.14260 / j e m d s / 2021 / 760$

How to Cite This Article:

Sood A, Sharma $R$, Katoch $R$, et al. Mortality pattern of in-born vs out-born neonates - comparative study of neonates admitted to special new born care units of a tertiary care hospital of hilly region of North India. J Evolution Med Dent Sci 2021;10(44):3757-3761, 10.14260/jemds/2021/760

Submission 27-09-2021, Peer Review 11-11-2021, Acceptance 17-11-2021, Published 30-11-2021.

Copyright (c) 2021 Ambika Sood et al. This is an open access article distributed under Creative Commons Attribution License [Attribution 4.0 International (CC BY 4.0)] 


\section{BACKGROUND}

India alone contributes to one-fifth of global live births and more than a quarter of neonatal deaths. It is further estimated that 130 million neonates are born each year and out of them, 4 million die within the first twenty-eight days of their life. Of the 25 million babies born in India, one million die every year, India alone contributes to $25 \%$ of neonatal mortality around the globe. ${ }^{1,2}$

Neonatal mortality accounted for maximum deaths in under five years with most of the mortalities occurring in developing countries. To address neonatal mortality comprehensively, it's important to understand the cause of death and risk factors for neonatal mortality. ${ }^{3}$

Neonatal deaths are also related to the quality of care a woman receives before and during her pregnancy and the quality of services available for the care of new-borns in the health facilities. The topmost causes of neonatal mortality worldwide include neonatal sepsis, infections, low birth weight, prematurity, birth asphyxia, birth trauma, congenital malformations, neonatal tetanus, diarrhoea, and pneumonia. The mortality risk is highest on the first day of life..$^{2-4}$

Goal 3 of sustainable development goals (SDG) targets to end preventable neonatal and under 5 deaths to a single digit by the year 2030 in India. With substantial heterogeneity in the pattern of mortality across the states of India, the interventions must be tailored at the state level. One of the interventions to promote the survival of new-borns is to create facilities for specialized maternal and neonatal care in peripheral hospitals. ${ }^{4,5}$

SNCU, as a part of new-born care, plays an important role in the reduction of neonatal mortality. Now, attention has been drawn to the need to improve the services at SNCUs. It is still unclear that whether this has been implemented uniformly across the country. Also, the facility-based studies in Himachal Pradesh are limited, creating a need to examine facility-based neonatal care in different settings. Such studies will provide information on the outcomes that are achievable at the different levels of care (inborn \& outborn) and highlight the modifiable factors that can improve outcome. . $^{3-5}$

Against this background, this study was undertaken to compare the mortality pattern among the outborn and inborn neonates admitted to the special new born care units (SNCUs) of IGMC \& KNH of Shimla district of Himachal Pradesh, India

\section{METHODS}

This retrospective study was conducted, between January 2016 and December 2020, for over 5 years, at the inborn and outborn SNCUs of Kamla Nehru Hospital and Indira Gandhi Medical College and Teaching Hospital. All newborns delivered at the tertiary care centre, Kamla Nehru Hospital (KNH), affiliated to IGMC, and admitted in the SNCU of KNH were considered as inborn neonates. All neonates delivered at the peripheral institutions and referred to the tertiary care centre for varied complaints and admitted to the SNCU of Indira Gandhi Medical College (IGMC) were considered as outborn neonates. All inborn and outborn neonates admitted at SNCUs of KNH and IGMC, Shimla, were included in the study, irrespective of gestation, birth weight and mode of delivery. Ethical clearance was obtained from the concerned authorities of the institutes.

\section{Statistical Analysis}

The data were collected from the record files of admitted neonates, compiled, and entered in MS Excel, and analysed using appropriate statistical tools in software Epi info V7 by applying the appropriate statistical test in terms of frequencies and percentage.

\section{RESULTS}

A total of 4018 neonates were admitted in the outborn SNCU of the paediatric ward of IGMC, Shimla while 6607 neonates were admitted in the inborn SNCU of Kamla Nehru Hospital Shimla. Among the outborn SNCU of the paediatric ward of IGMC, Shimla, the sex distribution was 2440 (60.73\%) males and 1578 (39.27\%) were females while in inborn SNCU of Kamla Nehru Hospital, Shimla, 3655 (55.32 \%) were males and 2952 (44.68\%) were females. (Table-1)

Among 4018 outborn neonates admitted in the SNCU of IGMC Shimla in 5 years, 3034 (75.51\%)were discharged after recovery, 305 (7.59\%) were referred to higher institutions, $372(7.90 \%)$ left against medical advice and 307 (7.64\%) died in SNCU while among 6607 inborn neonates, admitted in the SNCU of Kamla Nehru Hospital Shimla in 5 years, 5593 (84.65\%) were discharged after recovery, 313 (4.74\%) were referred to higher institutions, $335(5.07 \%)$ left against the medical advice and 366 (5.54 \%) died in SNCU (Table-1)

\begin{tabular}{|ccccc|}
\hline & \multicolumn{2}{c}{ Outborn (IGMC) } & \multicolumn{2}{c|}{ Inborn (KNH) } \\
Total & $\%$ & Total & $\%$ \\
\hline $\begin{array}{c}\text { Admission in the } \\
\text { unit }\end{array}$ & 4018 & 100.0 & 6607 & 100.0 \\
Male & 2440 & 60.73 & 3655 & 55.32 \\
Female & 1578 & 39.27 & 2952 & 44.68 \\
\multicolumn{4}{c}{ Outcome of Admission } \\
Discharge & 3034 & 75.51 & 5593 & 84.65 \\
Referral & 305 & 7.59 & 313 & 4.74 \\
LAMA & 372 & 9.26 & 335 & 5.07 \\
Died & 307 & 7.64 & 366 & 5.54 \\
\hline Table 1. The Trend of Neonatal Mortality in SNCU of Outborn vs Inborn \\
\hline \multicolumn{5}{c}{}
\end{tabular}

On retrospective analysis, we found that among the total of 307 outborn neonatal deaths in SNCU of IGMC Shimla, a maximum of 110 (35.83\%) were due to sepsis/pneumonia /meningitis, followed by $60(19.54 \%)$ due to respiratory distress syndrome, 50 (16.29\%) due to HIE/moderatesevere birth asphyxia, $36(11.73 \%)$ due to other causes, 24 $(7.84 \%)$ due to prematurity, $13(4.23 \%)$ due to unestablished causes, 12 (3.91\%) due to meconium aspiration syndrome, and $2(0.65 \%)$ due to major congenital malformation. Among the total of 366 inborn neonatal deaths in SNCU of KNH, a maximum of 115 (31.42\%) were due to respiratory distress syndrome, followed by 93 (25.41\%) due to sepsis/pneumonia/meningitis, $69(18.85 \%)$ due to HIE/moderate-severe birth asphyxia, 35 (9.56 \%) due to prematurity, 27 (7.38\%) due to other causes, 23 (6.28\%) due to major congenital malformation and $4(1.09 \%)$ due to Meconium aspiration syndrome. (Table-2) 


\begin{tabular}{|cccccc|}
\hline Reason of Mortality & $\begin{array}{c}\text { Outborn (IGMC) } \\
\text { Frequency }\end{array}$ & $\begin{array}{c}\text { Inborn (KNH) } \\
\text { Frequency }\end{array}$ & $\mathbf{\%}$ \\
Respiratory distress syndrome & 60 & 19.54 & 115 & 31.42 \\
Meconium aspiration syndrome & 12 & 3.91 & 4 & 1.09 \\
HIE/Moderate-severe Birth & 50 & 16.29 & 69 & 18.85 \\
Asphyxia & 110 & 35.83 & 93 & 25.41 \\
Sepsis/Pneumonia/Meningitis & 2 & 0.65 & 23 & 6.28 \\
Major congenital Malformation & 24 & 7.82 & 35 & 9.56 \\
Prematurity & 36 & 11.73 & 27 & 7.38 \\
Others & 13 & 4.23 & 0 & 0.00 \\
Cause not established & 307 & 100.00 & 366 & 100.00 \\
Total Mortality & Table 2. The Trend of Cause of Neonatal Mortality in SNCU of \\
\multicolumn{4}{c}{ IGMC \& KNH, Shimla } \\
\hline
\end{tabular}

When we analysed the data according to the total duration between admission and neonatal death in outborn SNCU of IGMC, Shimla, we found that maximum number of neonates 127 (41.37\%) stayed for 1-3 days in SNCU, 73 (23.78\%) for more than 7 days, 61 (19.87\%) for 4-7 days and $46(14.98 \%)$ for less than 1 day. Similarly, in inborn SNCU of Kamla Nehru Hospital Shimla, we found that maximum number of neonates 155 (42.35\%) stayed for 1-3 days in SNCU, 97 (26.50 \%) for 4-7 days, 58 (15.85 \%) for less than 1 day and $56(15.30 \%)$ for more than 7 days (Table-3)

\begin{tabular}{|ccccc|}
\hline \multirow{2}{*}{ Duration of Admission } & \multicolumn{2}{c|}{ Outborn (IGMC) } & \multicolumn{2}{c|}{ Inborn (KNH) } \\
Frequency & $\mathbf{\%}$ & Frequency & $\%$ \\
\hline$<1$ day & 46 & 14.98 & 58 & 15.85 \\
1-3 days & 127 & 41.37 & 155 & 42.35 \\
4-7 days & 61 & 19.87 & 97 & 26.50 \\
$>7$ days & 73 & 23.78 & 56 & 15.30 \\
Total death & 307 & 100.00 & 366 & 100.00 \\
\hline Table 3. Total Duration between Admission and Neonatal Death in \\
SNCU of IGMC \& KNH, Shimla \\
\hline
\end{tabular}

When we analysed the data according to age at death in outborn neonates SNCU of IGMC, Shimla, we found that maximum number of neonates 165 (53.75\%) were more than 7 days of age when they died, 129 (42.02\%) were of 1-6 days and only $13(4.23 \%)$ were less than a day when they died. Similarly, at inborn SNCU of Kamla Nehru Hospital Shimla, we found that maximum number of neonates, 236 (64.48\%) were of 1-6 days when died, 76 (20.77\%) were more than 7 days of age, while 54 (14.75\%) were of less than 1 day when they died. (Table-4)

\begin{tabular}{|ccccc|}
\hline & \multicolumn{2}{c|}{ Outborn (IGMC) } & \multicolumn{2}{c|}{ Inborn (KNH) } \\
Age at time of death & Total & $\mathbf{\%}$ & Total & $\%$ \\
$<1$ day & 13 & 4.23 & 54 & 14.75 \\
1-6 days & 129 & 42.02 & 236 & 64.48 \\
$>=7$ days & 165 & 53.75 & 76 & 20.77 \\
Total number & 307 & 100.00 & 366 & 100.00 \\
\hline Table 4. Age at Time of Death while at SNCU of IGMC \& & KNH, Shimla \\
\hline
\end{tabular}

According to weight at admission in SNCU of IGMC, Shimla, we found that outborn neonates 118 (38.44\%) had weight between 1500-2499 gm, 102 (33.22 \%) had > 2500 gm, 71 (23.13 \%) had between 1000-1499 gm while 16 (5.21 $\%)$ had weight < 1000 gm. Similarly, in SNCU of Kamla Nehru Hospital Shimla, we found that inborn neonates, 147 (40.16 $\%$ ) had weight between 1000-1499 gm, followed by 90 (24.59 \%) between 1500-2499 gm, 88 (24.04 \%) < $1000 \mathrm{gm}$ while $41(5.21 \%)$ had weight $>2500$ gm. (Table-5)

\begin{tabular}{|ccccc|}
\hline Weight at Admission & \multicolumn{2}{c}{ Outborn (IGMC) } & \multicolumn{2}{c|}{ Inborn (KNH) } \\
& Frequency & $\mathbf{\%}$ & Frequency & $\%$ \\
>=2500 gm & 102 & 33.22 & 41 & 11.20 \\
$1500-2499 \mathrm{gm}$ & 118 & 38.44 & 90 & 24.59 \\
$1000-1499 \mathrm{gm}$ & 71 & 23.13 & 147 & 40.16 \\
$<1000 \mathrm{gm}$ & 16 & 5.21 & 88 & 24.04 \\
Total number & 307 & 100.00 & 366 & 100.00 \\
\hline Table 5. Weight at Admission in SNCU of IGMC \&KNH, Shimla \\
\hline
\end{tabular}

We found that among the total of 307 outborn deaths at SNCU of IGMC Shimla, 131 (42.67\%) were preterm while 176 (57.33\%) had term birth. Similarly, at SNCU of KNH, among the total of 366 inborn deaths, 305 (83.3\%) were preterm while $61(16.7 \%)$ had term birth. (Table-6)

\begin{tabular}{|ccccc|}
\hline \multirow{2}{*}{ Total Gestation } & \multicolumn{2}{c}{ Outborn (IGMC) } & \multicolumn{2}{c|}{ Inborn(KNH) } \\
& Frequency & $\%$ & Frequency & $\%$ \\
\hline Term & 176 & 57.33 & 61 & 16.7 \\
Preterm & 131 & 42.67 & 305 & 83.3 \\
Total number & 307 & 100.00 & 366 & 100.0 \\
\hline \multicolumn{4}{c}{ Table 6. Gestation Period at Admission in SNCU of } \\
IGMC \& $\mathbf{K N H}$, Shimla \\
\hline
\end{tabular}

\section{DISCUSSION}

This study highlights the differences and similarities between neonates admitted at outborn SNCU of IGMC Shimla with the inborn SNCU of KNH Shimla for 5 years.

Among the 4018 outborns admitted in SNCU of the paediatric ward of IGMC, Shimla, 2440 (60.73\%) were males and 1578 (39.27\%) were females while in 6607 inborns admitted at SNCU of Kamla Nehru Hospital Shimla, 3655 (55.32\%) were males and 2952 (44.68 \%) were females. The observed difference between the number of admissions in these SNCUs was possible because most of the patients from the KNH were inborns while IGMC received mostly referral outborn cases from all 12 districts of Himachal Pradesh.

Also, the majority of the admissions was males as compared to females in both SNCUs. The male predominance is due to the biological vulnerability of the male gender and may be due to the preference of male children in almost every society in India. Similar findings have also been reported from various studies conducted in different parts of India. ${ }^{6-12}$

In the present study, at SNCU of IGMC Shimla, 307 outborns $(7.64 \%)$ died. While in the SNCU of Kamla Nehru Hospital Shimla, 366 inborns (5.54\%) died over 5 years. The higher numbers reported at IGMC could be due to more highrisk admissions. Similarly, one would have expected higher mortality due to the referral of high-risk babies from all 12 districts of Himachal Pradesh.

In the current study, at outborn SNCU of IGMC Shimla, a maximum of 110 (35.83 \%) were due to Sepsis/ Pneumonia / Meningitis, followed by $60(19.54 \%)$ due to respiratory distress syndrome and $50(16.29 \%)$ due to HIE/Moderatesevere Birth Asphyxia, while in inborn SNCU of KNH, maximum deaths 115 (31.42\%) were due to Respiratory distress syndrome, followed by 93 (25.41\%) due to Sepsis/Pneumonia/Meningitis and 69 (18.85\%) due to HIE/Moderate-severe Birth Asphyxia. A similar pattern of causes of neonatal mortality has been reported by studies conducted in various parts of India and reported by ICMR \& JIPMER.6-14

A reduction in the cases of neonatal sepsis can be achieved through clean delivery practices, intrapartum antibiotics, handwashing by caregivers and health professionals, early initiation of breastfeeding, maternal and other forms of neonatal antisepsis. By adopting these preventive measures, we can reduce the workload, shorten the duration of stay, avoid cross infection and reduce neonatal mortality.2,15,16 
The present study also highlights the need to prevent sepsis, pneumonia, respiratory infection and support respiration. These could be prevented by improving important preventive services like quality antenatal care, early recognition of danger signs, timely referral when the signs of respiratory distress occur and prompt intervention at the health facilities. ${ }^{2,10,11}$

Focus on intranatal care and new born resuscitation within the "golden minute" would help to prevent birth asphyxia. These SNCUs should be strengthened with the availability of CPAP (Continuous Positive Airway Pressure) and surfactants to manage premature new-borns thereby reducing RDS related deaths. Capacities of the health staff of the delivery room need to be enhanced to provide skilled maternal and essential new born care. In addition, research to determine the epidemiology of neonatal sepsis, their causative organisms, and their sensitivity patterns to antimicrobials \& cross infectivity patterns is also desirable. ${ }^{10,11,16}$

In our study, at outborn SNCUs of IGMC, Shimla, maximum number of neonates 127 (41.37\%) stayed for a duration of 1-3 days in SNCU also in the inborn SNCU of Kamla Nehru Hospital Shimla, maximum neonates 155 (42.35 $\%)$ stayed for 1-3 days. At SNCU of IGMC, Shimla, most of the outborn neonates 165 (53.75\%) were more than 7 days of age when they died while in case of inborn SNCU of Kamla Nehru Hospital Shimla, most of the neonates 236 (64.48 \%) were of 1-6 days when they died. These findings reflect that these referring units have spent time trying to stabilize the neonates, but they could not bear the fruit.

In the present study, SNCU of IGMC, Shimla, of the outborn neonates 118 (38.44\%) had weight between 15002499 gm, while, in SNCU of Kamla Nehru Hospital Shimla, most of the inborn neonates 147 (40.16\%) weighed between 1000-1499 gm. In our study, at SNCU of IGMC Shimla, 131 (42.67\%) outborns were preterm while at SNCU of KNH, 305 $(83.3 \%)$ inborns were preterm. Further studies on risk factors for prematurity and low birth weight are needed. To improve the outcome in case of prematurity and low birth weight, more skills in resuscitation and stabilizing neonates in the first few days of admission are required in health care staff of SNCUs including respiratory support.2,11,16

\section{CONCLUSIONS}

According to the present study, neonatal sepsis, birth asphyxia and RDS are the leading causes of mortality in the new-borns admitted in both the SNCUs. Despite, many advances in facility-based neonatal care in Himachal Pradesh, the above factors continue to be the leading causes of mortality in neonates. The majority of these mortalities can be prevented by improving antenatal care, maternal health, recognition of danger signs, well-equipped facilities, skilled health care providers, timely referral, a strong link between communities and health facilities, and prompt intervention at the health facilities can reduce the neonatal mortality rate.

The study also highlights the need to understand the "Levels of delay" (delay in decision making, delay in accessing transport and delay to reach the facility) and the referral pathway taken to reach the appropriate health facilities. Present data point to equipping the SNCUs with staff having the skills to manage birth asphyxia and preterm delivery and provide adequate care to the neonates, through prompt identification, stabilization, and appropriate referral, and to improve the understanding of neonatal mortality by gestation months to develop facility \& community-based interventions to better manage the new-borns.

\section{Limitations}

This study has some limitations. Firstly, this was a hospitalbased retrospective study, the cause of death was determined using the data available in case record sheets. Second, the results from this study cannot be a complete reflection of the problem in the community. Therefore, multicentric, prospective studies including major centres providing neonatal care in this hilly region will provide a better idea of neonatal survival.

Data sharing statement provided by the authors is available with the full text of this article at jemds.com.

Financial or other competing interests: None.

Disclosure forms provided by the authors are available with the full text of this article at jemds.com.

\section{REFERENCES}

[1] Sankar MJ, Neogi SB, Sharma J, et al. State of newborn health in India. J Perinatol 2016;36(s3):S3-8.

[2] Uppal K, Ashwani N, Jeelani K, et al. Profile of neonatal mortality in SNCU district hospital. Galore International Journal of Health Sciences \& Research 2019;4(1):6-8,

[3] Dandona R, Kumar GA, Bhattacharya D, et al. Distinct mortality patterns at $0-2$ days versus the remaining neonatal period: results from population-based assessment in the Indian state of Bihar. BMC Med 2019;17(1):140.

[4] Sharma AK, Gaur A. Profile of neonatal mortality in special newborn care unit of tertiary care hospital. Int J Contemp Pediatr 2019;6(6):2319-25.

[5] Tette EMA, Nartey ET, Nuertey BD, et al. The pattern of neonatal admissions and mortality at a regional and district hospital in the Upper West Region of Ghana; a cross sectional study. PLoS One 2020;15(5):e0232406.

[6] Babu MC, Prakash PS, Prasanna CL. Neonatal morbidity and mortality patterns of babies admitted in SNCU @ ACSR Government Medical College, Nellore, Andhra Pradesh. J Evol Med Dent Sci 2018;7(02):203-6.

[7] Kumar MK, Thakur SN, Singh BB. Study of the morbidity and the mortality patterns in the neonatal intensive care unit at a tertiary care teaching hospital in Rohtas District, Bihar, India. J Clin Diagnos Res 2012;6(2):282-5.

[8] Rashid A, Ferdous S, Chowdhury T, et al. The morbidity pattern and the hospital outcome of the neonates who were admitted in a tertiary level hospital in Bangladesh. Bangladesh J Child Health 2003;27:10-3.

[9] ICMR Young Infant Study Group. Age profile of neonatal deaths. Indian Pediatr 2008;45(12):991-4.

[10] Mundlod S, Thakkarwad S. Mortality profile and outcome analysis in level two SNCU in tribal medical college district Adilabad Telangana. Int J Med Paediatr Oncol 2019;5(4):125-8. 
[11] Som M, Nayak C, Padhi BK, et al. Patterns of morbidity among newborns admitted in SNCUs of Odisha, India. Int J Health Sci Res 2018; 8(9):10-19.

[12] Modi R, Modi B, Patel JK, et al. Study of the morbidity and the mortality pattern in the neonatal intensive care unit at a tertiary care teaching Hospital in Gandhinagar District, Gujarat, India. J Res Med Den Sci 2015;3(3):20812.

[13] Sridhar PV, Thammanna PS, Sandeep M. Morbidity pattern and hospital outcome of neonates admitted in a tertiary care teaching hospital, Mandya. Int J Sci Stud 2015;3(6):126-9.
[14] Lawn JE, Cousens S, Zupan J. 4 million neonatal deaths: When? Where? Why? Lancet 2005;365(9462):891-900.

[15] Neogi SB, Malhotra S, Zodpey S, et al. Assessment of special care newborn units in India. J Health Popul Nutr 2011;29(5):500-9.

[16] Jena D, Tripathy RM, Pradhan S, et al. Assessment of socio-clinical profile of neonates admitted in sick neonatal care unit of tertiary care hospital: Odisha. Int J Res Med Sci 2017;5(9):4077-81. 